\title{
Targeted antibody-mediated depletion of murine CD19 CAR T cells permanently reverses B cell aplasia
}

\author{
Paulina J. Paszkiewicz, ${ }^{1,2}$ Simon P. Fräßle, ${ }^{1,2}$ Shivani Srivastava, ${ }^{3}$ Daniel Sommermeyer, ${ }^{3}$ Michael Hudecek, ${ }^{4}$ Ingo Drexler, ${ }^{5}$ \\ Michel Sadelain, ${ }^{6}$ Lingfeng Liu, ${ }^{3}$ Michael C. Jensen, ${ }^{3,7,8}$ Stanley R. Riddell, ${ }^{2,3,7}$ and Dirk H. Busch ${ }^{1,2,9}$ \\ "Institute for Medical Microbiology, Immunology and Hygiene, Technische Universität München (TUM), Munich, Germany. ${ }^{2}$ Focus Group "Clinical Cell Processing and Purification," Institute for

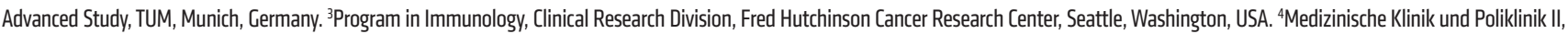 \\ Universitätsklinikum Würzburg, Würzburg, Cermany. Institute for Virology, Universitätsklinikum Düsseldorf, Heinrich-Heine Universität, Düsseldorf, Germany. ${ }^{6}$ Center for Cell Engineering, Memorial Sloan \\ Kettering Cancer Center, New York, New York, USA. 'Department of Medicine, University of Washington, Seattle, Washington, USA. ${ }^{8}$ Seattle Children's Research Institute, Ben Towne Center for Childhood \\ Cancer Research, Seattle, Washington, USA. ${ }^{9}$ National Center for Infection Research (DZIF), Munich, Germany.
}

\begin{abstract}
The adoptive transfer of T cells that have been genetically modified to express a CD19-specific chimeric antigen receptor (CAR) is effective for treating human B cell malignancies. However, the persistence of functional CD19 CAR T cells causes sustained depletion of endogenous CD19+ B cells and hypogammaglobulinemia. Thus, there is a need for a mechanism to ablate transferred T cells after tumor eradication is complete to allow recovery of normal B cells. Previously, we developed a truncated version of the epidermal growth factor receptor (EGFRt) that is coexpressed with the CAR on the T cell surface. Here, we show that targeting EGFRt with the IgG1 monoclonal antibody cetuximab eliminates CD19 CAR T cells both early and late after adoptive transfer in mice, resulting in complete and permanent recovery of normal functional B cells, without tumor relapse. EGFRt can be incorporated into many clinical applications to regulate the survival of gene-engineered cells. These results support the concept that EGFRt represents a promising approach to improve safety of cell-based therapies.
\end{abstract}

\section{Introduction}

Adoptive transfer of genetically engineered $\mathrm{T}$ cells is a rapidly emerging area in cell-based cancer therapy. The most advanced application is the use of CD19 chimeric antigen receptor (CAR) $\mathrm{T}$ cells, which has demonstrated antitumor efficacy in patients with refractory B cell malignancies including acute lymphoblastic leukemia (ALL) and non-Hodgkin's lymphoma (1-3). CD19 is upregulated at the early stages of B cell development and expressed throughout the B cell lineage; only after differentiation to plasma cells is CD19 expression lost (4). Thus, an unavoidable side effect of transferring CD19 CAR T cells is the depletion of endogenous B cells, which, if sustained, results in hypogammaglobulinemia and places the patient at risk of life-threatening infections (5).

Since CD19 CAR T cell therapy can lead to complete and apparently durable tumor remissions in B cell malignancies, and CARs specific for molecules on solid tumors are being developed (6), there is a growing need to develop strategies to treat long-term side effects caused by CAR T cells. Available techniques to selectively eliminate adoptively transferred $\mathrm{T}$ cells in vivo are based, for example, on genetic integration of herpes simplex virus thymidine kinase (HSV-TK) or inducible caspase-9 (iCasp9) $(7,8)$. HSV-TK efficiently ablates cycling cells upon treatment with substrates (like

Authorship note: P.J. Paszkiewicz and S.P. Fräßle contributed equally to this work. D.H. Busch and S.R. Riddell share senior authorship.

Conflict of interest: S.R. Riddell, M. Sadelain, M.C. Jensen, and D.H. Busch hold shares in Juno Therapeutics.

Submitted: September 24, 2015; Accepted: September 8, 2016.

Reference information: J Clin Invest. 2016;126(11):4262-4272. doi:10.1172/JCI84813. ganciclovir); however, immunogenicity of the viral TK can result in premature rejection of TK-expressing T cells (9), which limits its clinical suitability $(10,11)$. Introduction of the non-immunogenic iCasp9 into donor lymphocyte infusions showed promising results in hematopoietic stem cell recipients to treat graft versus host disease (GVHD) caused by the transferred T cells (8). Here, efficient in vivo depletion is achieved by infusion of the dimerizer AP1903 that initiates cell apoptosis via activation of iCasp9. The limited availability of the dimerizer for clinical use currently constrains the broader application of this suicide mechanism. Furthermore, it is not yet known how efficient iCasp9-mediated cell depletion really is; in the GVHD setting it may be sufficient just to reduce the total number of pathogenic cells. Sustained long-term and complete depletion will likely be necessary for achieving B cell recovery upon CD19 CAR T cell therapy, since it has been shown that even very small numbers of surviving memory $\mathrm{T}$ cells with stem cell-like properties are capable of restoring a functional immune response within a short period of time (12).

Antibody-dependent depletion mechanisms can mediate highly efficient $\mathrm{T}$ cell elimination by recruiting endogenous cytolytic effector pathways, including antibody-dependent cellular cytotoxicity and complement-dependent cytotoxicity. These approaches require a cell surface molecule that is coexpressed with the tumor-targeting receptor. For example, T cells have been genetically engineered to express the full-length CD20 receptor or a construct comprising the target epitope of the CD2O-specific antibody rituximab to mediate in vivo lysis of $\mathrm{T}$ cells using rituximab $(13,14)$. As rituximab treatment inevitably leads to depletion of endogenous $\mathrm{CD}^{2} \mathrm{O}^{+}$B cells, CD20 is not a preferable safety 

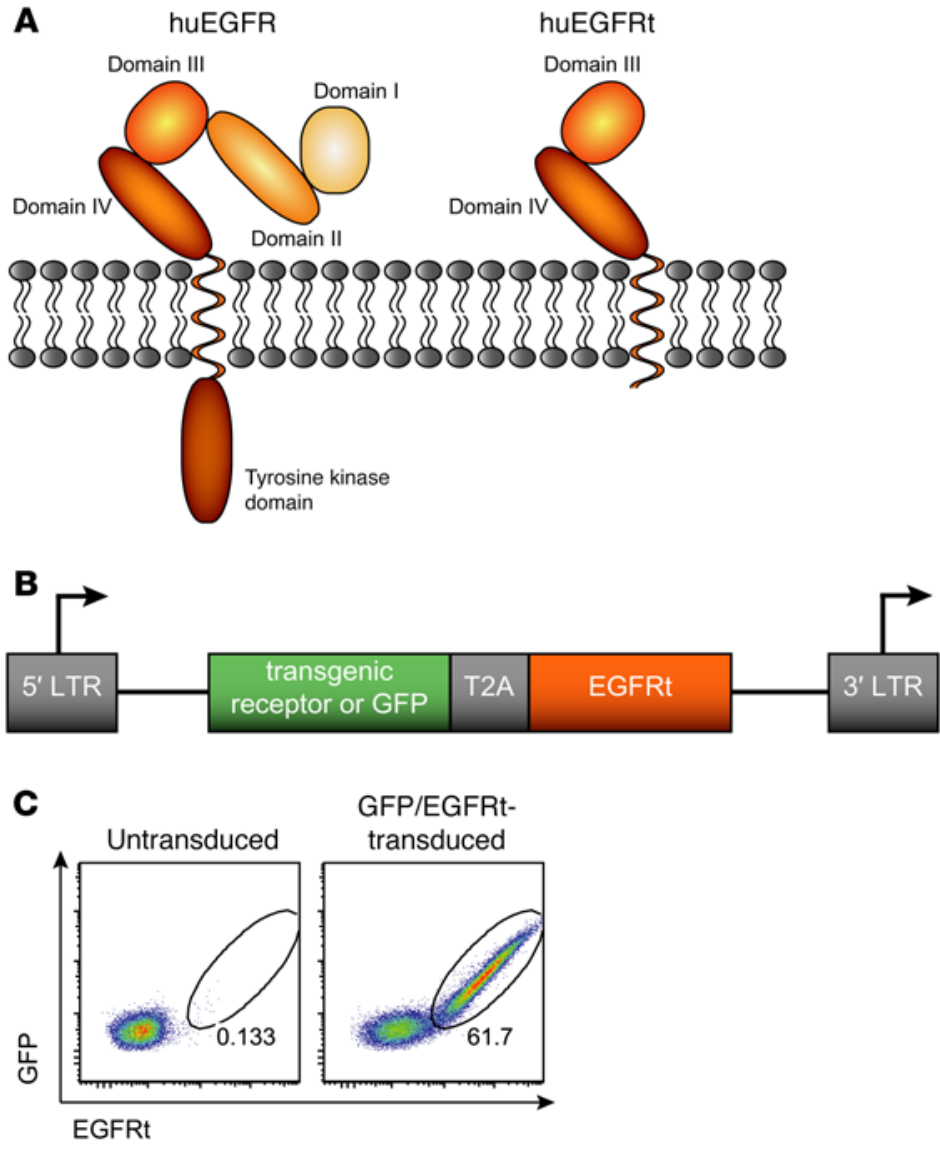

marker to facilitate reconstitution of the B cell compartment upon CD19 CAR T cell therapy. In an alternative approach, a Myc-tag has been directly tethered to the recombinant antigen receptor, which allows in vivo targeting by a depleting anti-Myc antibody (15). Albeit effective, this strategy is limited because there is no clinically approved antibody available that is specific to c-Myc. Also, regarding "completeness" of depletion, conclusive data are not yet available for both CD20 and c-Myc.

We developed a non-immunogenic cell surface EGFR-like molecule as a target for cetuximab, a clinically available IgG1 mAb. The human EGFR molecule was truncated in the extracellular domain to eliminate binding of endogenous ligands such as EGF and in the intracellular kinase domain to exclude signaling (16). This functionally inert truncated EGFR (EGFRt) can be coexpressed with any recombinantly expressed receptor on the cell surface and might serve as a cell-specific target for in vivo cell ablation.

In this report, we examined the utility of EGFRt as a target for antibody-mediated depletion of CD19 CAR T cells in a clinically relevant mouse model. We demonstrate that cetuximab efficiently and specifically eliminates CAR T cells expressing the EGFRt marker, which resulted in long-term numerical and functional reversal of B cell aplasia.

\section{Results}

Coexpression of functionally inert EGFRt on the T cell surface. The truncated EGF receptor (EGFRt) has previously been introduced as a suitable cell surface marker for tracking, selection, and depletion of engineered T cells (16). The EGFRt is derived from the
Figure 1. Truncated EGFR implemented into a T2A-containing gene expression vector allows for stringent coexpression of recombinant proteins. (A) Schematic representation of the human EGFR (huEGFR) illustrates the structure of the WT EGFR, including 4 extracellular domains and an intracellular tyrosine kinase domain. The truncated form of the EGFR (huEGFRt) lacks domains I and II and most of the cytoplasmic region of the fulllength EGFR. (B) The EGFRt can be included into gene expression vectors, e.g., encoding for a transgenic receptor or GFP, including a T2A linker for coexpression. LTR, long terminal repeats; T2A, Thosea asigna virus $2 \mathrm{~A}$ peptide sequence. (C) Mouse splenocytes were transduced with a GFP/EGFRt gene construct. EGFR expression was detected with a biotinylated anti-EGFR mAb and streptavidin-phycoerythrin on day 4 after transduction. Cells were pregated on living lymphocytes, and numbers indicate the percentage of GFP/EGFRt ${ }^{+}$cells. Similarly stained untransduced cells served as a negative control. human full-length EGFR and truncated to ensure nonfunctionality for physiological EGFR activities (Figure 1A). The extracellular domains I and II are removed to abrogate binding to any endogenous ligands, such as EGF or TGF- $\alpha$. In addition, intracellular tyrosine kinase domains are deleted to prevent receptor signaling events. Via a GM-CSF receptor leader peptide of 22 amino acids (aa), this residual EGFRt sequence spanning aa 334-668 of the mature WT molecule is efficiently expressed on the cell surface. Because of its relatively small size of 357 aa, the leader/EGFRt construct can be easily implemented into most gene expression vectors, and genes encoding for a transgenic receptor (or GFP as an experimental tracer molecule) can be simultaneously and in an equimolar fashion expressed together with EGFRt by the Thosea asigna virus $2 \mathrm{~A}$ (T2A)-ribosomal skip sequence (Figure 1B). In order to demonstrate coexpression, mouse splenocytes were genetically engineered by retroviral transduction to express the GFP/EGFRt gene construct. And indeed, expression levels of GFP and the EGFRt were found to be tightly correlated, as shown by the detection of double-positive cells upon surface staining with an EGFR-specific antibody in combination with GFP expression levels (Figure 1C).

EGFRt is stably expressed on T cells in vivo and can be targeted with anti-EGFR $m A b$. Since protein coexpression in vitro does not necessarily predict stable long-term coexpression in vivo, we analyzed the robustness of EGFRt surface staining on adoptively transferred engineered T cells in vivo. We transduced Thy1.1 $1^{+}$OT-I $\mathrm{T}$ cell receptor (TCR) transgenic $\mathrm{T}$ cells with a retroviral construct encoding GFP/EGFRt, and purified the fraction of GFP/EGFRt 
A

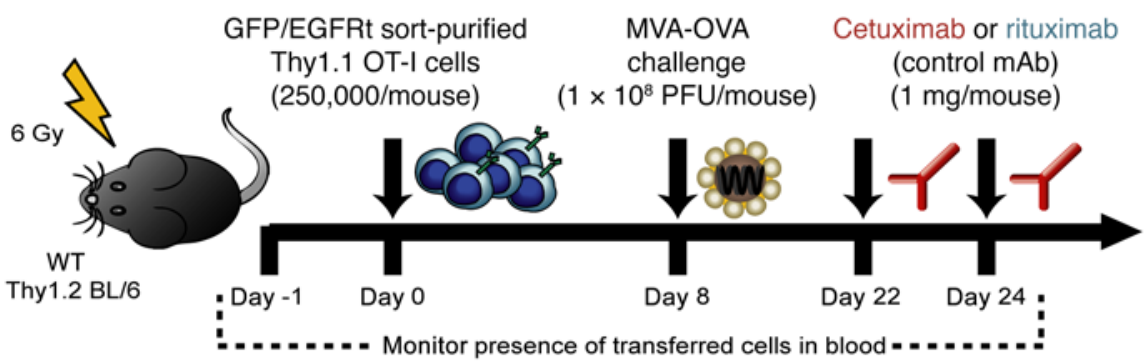

B

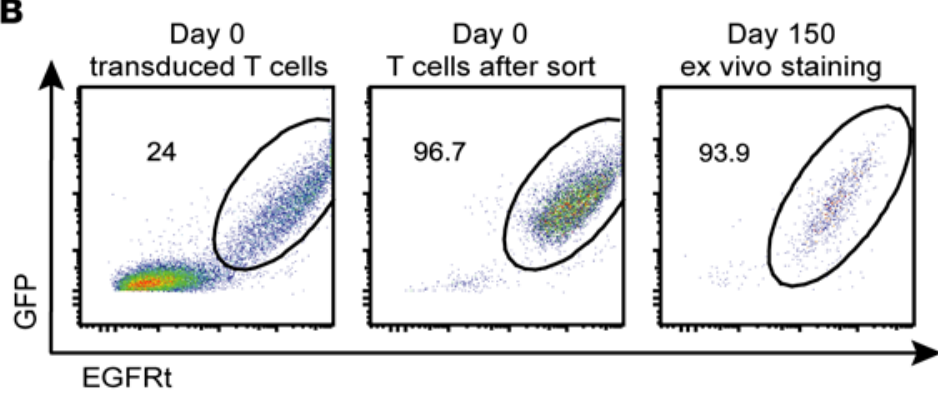

C

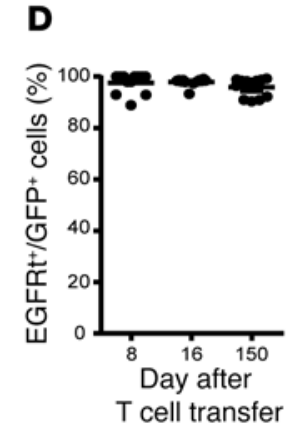

E

Cetuximab group

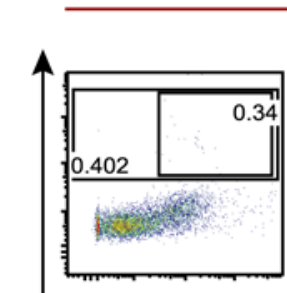

Pregated on

Rituximab group
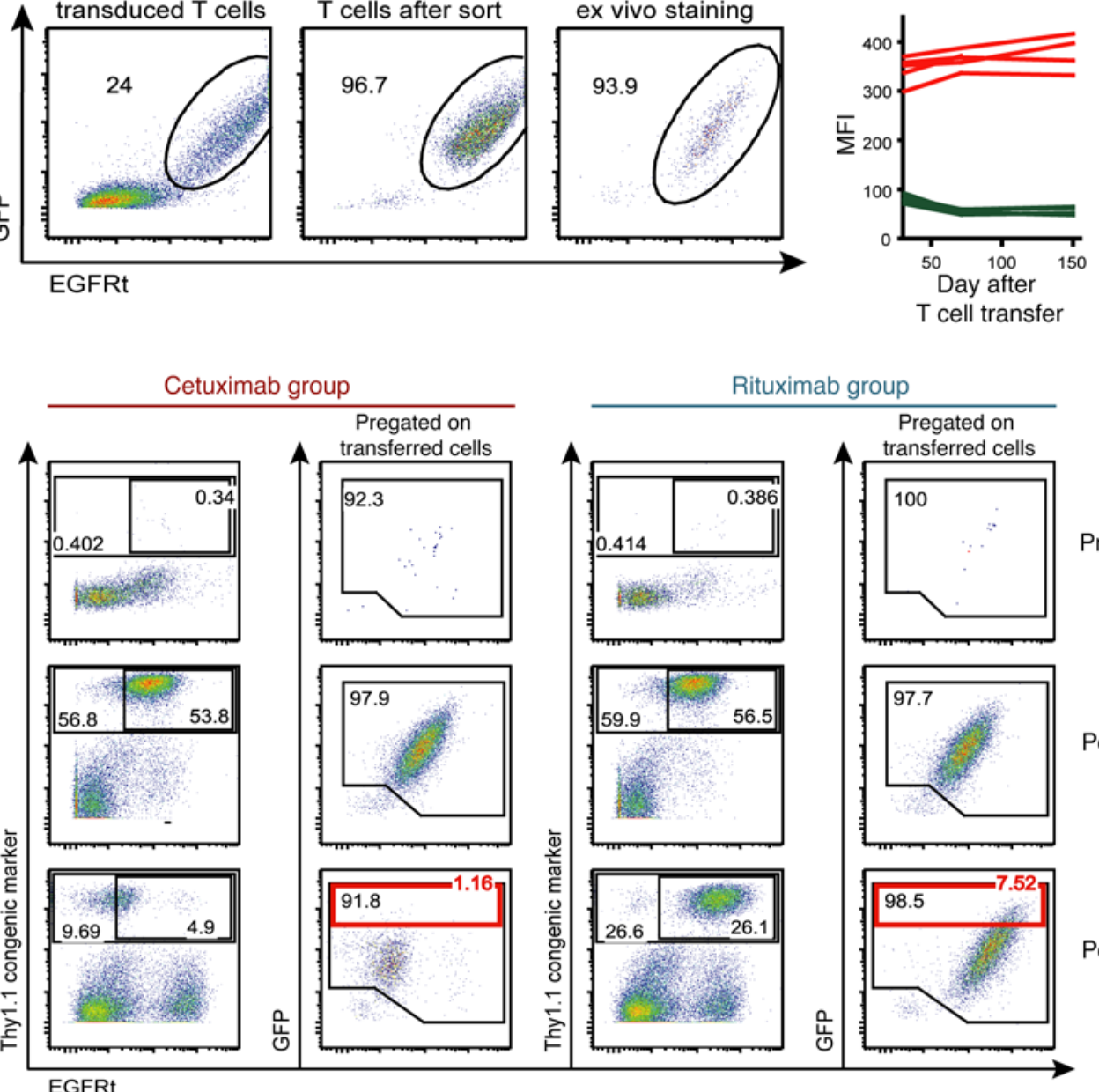

Pre-MVA-OVA

Post-MVA-OVA
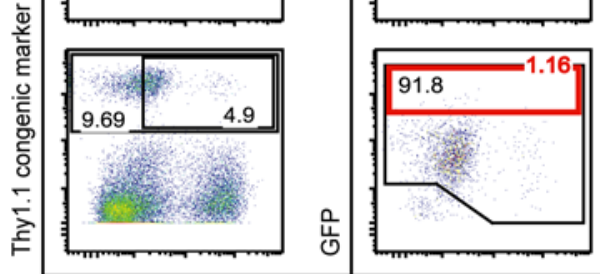

EGFRt

$\mathbf{F}$

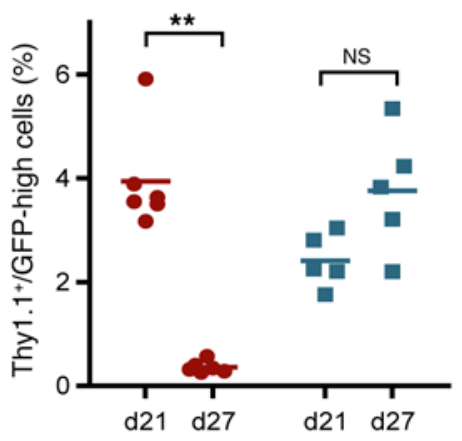

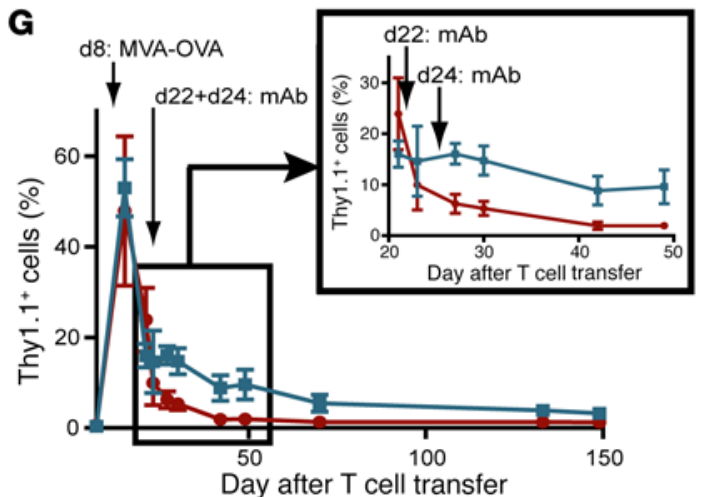


Figure 2. Recombinant surface EGFRt is stably expressed over time and can be used for antibody-mediated $\mathrm{T}$ cell depletion in vivo. (A) Experimental layout of depletion experiments in the OT-I mouse model. (B) GFP/ EGFRt-transduced Thy $1.1^{+}$mouse splenocytes were sort-purified using EGFR Fab Streptamers, subsequently transferred into sublethally irradiated mice, and then tracked over time in the blood. Coexpression of GFP and EGFRt is shown for transduced cells before (left) and after sorting on the day of T cell transfer (middle; day 0 ), as well as in a blood sample on day 150 after infusion (right). Cells were stained with the EGFR-specific mAb, and the frequency of GFP/EGFRt double-positive cells among living Thy1.1 $1^{+}$ cells is shown for samples obtained from representative animals. (C) Blood samples were obtained over 150 days after transfer of GFP/EGFRt-sort-purified cells. The level of GFP expression was acquired and EGFR expression was detected with EGFR-specific mAbs. The GFP and EGFR expression levels of living Thy $1.1^{+}$cells, as represented by the mean fluorescence intensity (MFI), were plotted over time for 4 individual animals. (D) Summary of flow cytometry analysis of GFP+/EGFRt ${ }^{+}$double-positive cells pregated on Thy1.1 $1^{+}$EGFRt ${ }^{+}$cells for 3 different time points. $n=12$. (E) Two hundred fifty thousand GFP/EGFRt ${ }^{+}$Thy $1.1^{+}$OT-I cells were transferred into WT C57BL/6 mice. Blood samples were obtained before (pre-MVA-OVA) and after challenge with MVA-OVA (post-MVA-OVA), as well as after infusions of cetuximab or rituximab (post-mAb). The Thy1.1 congenic marker and the EGFRt were detected with Thy1.1- and EGFR-specific mAbs. Gates were set on all living transferred Thy $1.1^{+}$cells (larger gate) and EGFRt ${ }^{+}$Thy $1.1^{+}$cells (smaller gate), and numbers indicate respective frequencies. GFP versus EGFR staining of the Thy1.1+ pregated cells is also shown with an additional gate on CFP-high cells in red. A representative sample of the cetuximab and rituximab group is shown. (F) Frequencies of Thy1.1+ GFP-high cells for blood samples acquired on day 21 and day 27 (see scheme in $\mathbf{A}$ ) from the cetuximab-treated (Ctx) and rituximab-treated (Rtx) mouse groups. Ctx group: $n=6$; Rtx group: $n=5$. Two-tailed Mann-Whitney test was used for statistical significance. ${ }^{* *} P \leq 0.005$. (C) Time course for the Thy $1.1^{+}$cell frequencies. Inset shows section from days $21-49$ and a different $y$ axis scale. Means \pm SD are plotted.

double-positive T cells by flow cytometry-guided cell sorting. Sublethally irradiated WT (Thy1.1-) C57BL/6 mice received 250,000 Thy1.1 $1^{+}$GFP/EGFRt ${ }^{+} \mathrm{T}$ cells per recipient, and were subsequently challenged with a modified vaccinia virus Ankara-based vector encoding ovalbumin (MVA-OVA) to expand the transferred T cells and establish readily detectable and durable memory populations (Figure 2A). T cell persistence and EGFRt expression levels were monitored by frequent analysis of blood samples. As shown in Figure 2, B-D, EGFRt surface expression was found to be highly stable over time and mirrored the coexpression of EGFRt and GFP in transduced cells before transfer and throughout 150 days of in vivo persistence. There was no evidence for any segregation into single-positive subsets, which would have indicated asymmetric surface expression that could complicate in vivo targeting of all transferred T cells via EGFR-specific antibodies.

Encouraged by the stable long-term coexpression of GFP/ EGFRt-transduced effector and memory $\mathrm{T}$ cells, we evaluated whether administration of the EGFR-specific mAb cetuximab can specifically eliminate EGFRt-expressing T cells in vivo. Groups of Thy $1.2^{+}$recipient mice received highly enriched EGFRt ${ }^{+}$Thy $1.1^{+}$ OT-I T cells, which were subsequently expanded to large numbers in vivo by MVA-OVA vaccination. Two weeks later, 1 group of mice received 2 doses of $1 \mathrm{mg}$ cetuximab, and a control group was treated similarly with rituximab. Transferred Thy $1.1^{+}$cell populations were readily detectable in all groups after MVA-OVA challenge. However, already during the first days after cetuximab admin- istration, GFP'Thy1.1+ OT-I T cells rapidly decreased, with complete disappearance of the subset of OT-I T cells that expressed the highest levels of GFP, although some cells with low-level GFP expression remained detectable (Figure 2, E and F). In the rituximab control group, the numbers of GFP'Thy1.1. OT-I T cells were unchanged, and the distribution of GFP-high- and GFP-lowexpressing cells remained unaffected. It is important to note that the ex vivo staining for EGFR shown in Figure 2 does not necessarily reflect the true EGFRt surface expression in all groups, because binding of the detection antibody (which was also derived from cetuximab) could be blocked by in vivo antibody binding to the target cell surface in mice treated with cetuximab (Figure 2E). In control experiments we could indeed demonstrate reduced EGFR staining intensity if EGFRt ${ }^{+}$cells were preincubated with cetuximab (Supplemental Figure 1; supplemental material available online with this article; doi:10.1172/JCI84813DS1). Even more convincingly, we observed a similar blocking effect when preincubating EGFRt-expressing $\mathrm{T}$ cells with plasma derived from mice recently treated with cetuximab, and using Fc-specific staining reagents we could directly visualize surface-bound antibodies. Therefore, it was very important to include GFP as an additional detection and expression marker.

We monitored the frequencies of transferred Thy1.1+ OT-I cells in the blood and observed peak expansion on day 8 after MVA-OVA challenge and decreasing cell numbers during the contraction phase of virus infection (Figure 2G). After antibody infusions, cetuximab clearly depleted a large fraction of GFP/EGFRt ${ }^{+}$ cells, while transferred cells were maintained at higher levels in the rituximab group. In cetuximab-treated mice, the frequency of transferred cells further decreased until day 150. In addition to blood, we assessed cell persistence in secondary lymphoid organs, i.e., spleen and lymph nodes, and the lung on day 238 after T cell infusion (Supplemental Figure 2). We observed that GFP-highexpressing cells were efficiently depleted by cetuximab in all analyzed organs. At this late time point, the EGFR staining was no longer blocked in the cetuximab group, indicating that cetuximab had been cleared from the blood.

In summary, these depletion studies with sorted GFP/EGFRt ${ }^{+}$ OT-I cells demonstrate that cetuximab mediates efficient in vivo depletion of T cells that express high levels of EGFRt. Some T cells with low GFP/EGFRt expression survive the cetuximab treatment or get depleted with substantially slower kinetics.

Expression level of EGFRt determines the kinetics of in vivo depletion of transduced $T$ cells. In order to better understand how the level of EGFRt expression on engineered $\mathrm{T}$ cells affects the kinetics and efficiency of cetuximab-mediated in vivo depletion, we transduced OT-I cells with the GFP/EGFRt construct and subsequently sorted the cells based on their level of GFP/EGFRt expression (Figure $3 \mathrm{~A})$. We then adoptively transferred $1 \times 10^{6} \mathrm{CD} 45.1^{+}$EGFRt-high, EGFRt-low, or mock-transduced cells into separate groups of sublethally irradiated WT C57BL/6 mice. As described before, MVAOVA vaccination was performed to expand OT-I T cells in vivo, followed by 2 infusions of cetuximab. Analysis of the frequencies of $\mathrm{GFP}^{+}$cells (pregated on the transferred CD45.1 $1^{+}$cells) indicated that cells with high GFP/EGFRt expression were depleted by cetuximab to numbers below the detection limit, whereas low-expressing cells were still detectable (Figure 3B). To better quantify these 
A

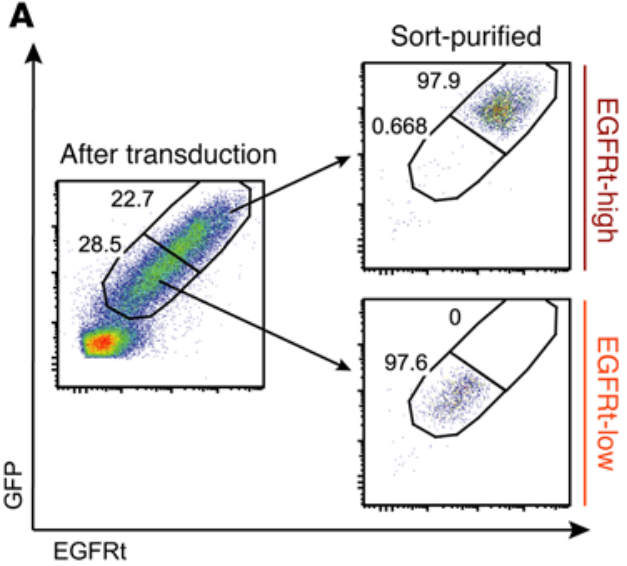

d16: mAb

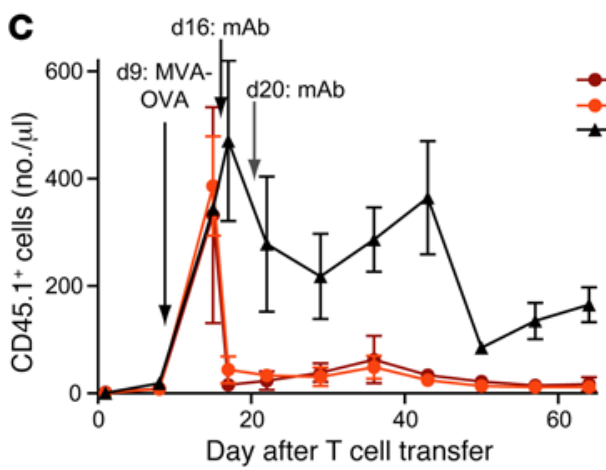

D

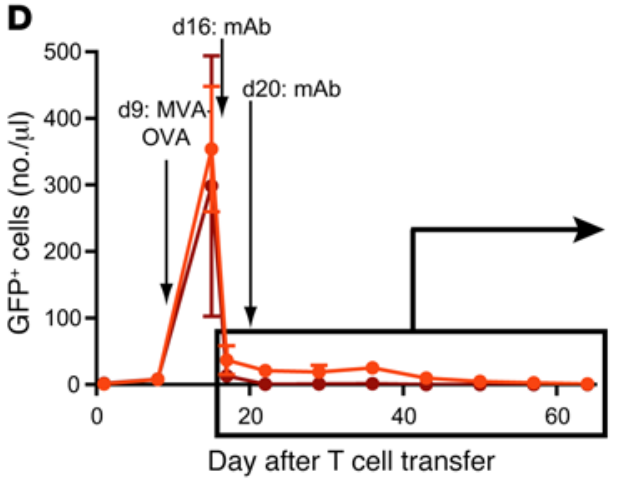

B
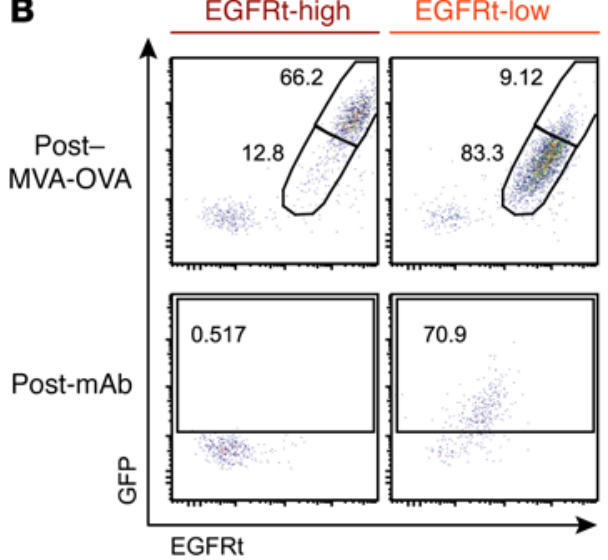

GFR-high EGFR-low Mock

\section{E}

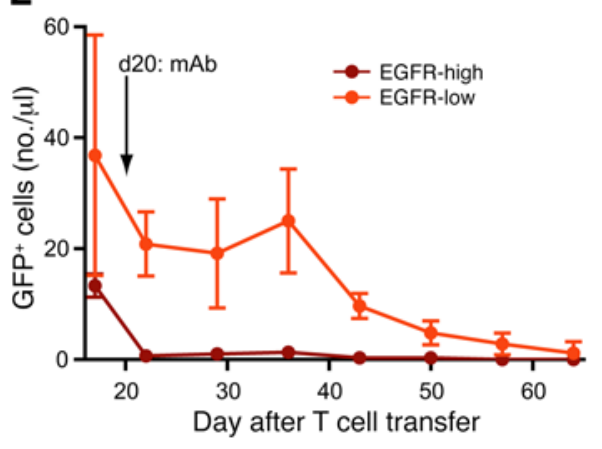

Figure 3. T cells enriched for high EGFRt expression are efficiently depleted by cetuximab in vivo. (A) GFP/EGFRt-transduced CD45.1 $1^{+}$OT-I cells were sorted into GFP/EGFRt-high and -low populations using EGFR Fab Streptamers on day 5 after transduction. Unsorted and FACS-sorted samples were stained with an EGFR-specific $m A b$. Numbers indicate frequencies of GFP/EGFRt-high (top right gate) and GFP/EGFRt-low (bottom right gate) cells. (B) $1 \times 10^{6}$ sorted OT-I cells were transferred into separate mouse groups, called EGFRt-high and EGFRt-low, and expanded in vivo by MVA-OVA vaccination. Cetuximab was administered to both mouse groups. Blood samples were obtained after MVA-OVA challenge and after $\mathrm{mAb}$ infusions. GFP versus EGFR staining of CD45.1-pregated cells is shown. Frequencies of GFP/EGFRthigh and -low cells are indicated in the top row, frequencies of GFP+ ${ }^{+}$cells in the bottom row. (C) Blood samples were obtained from 2 mouse groups that received EGFRt-high or -low cells; time points of MVA-OVA challenge and cetuximab infusions are indicated. The control group received mock-transduced OT-I cells and similar infusions. Transferred cells among total living lymphocytes were detected with a CD45.1-specific mAb. Number of CD45.1 $1^{+}$cells per microliter blood was plotted over time for each mouse group. (D) Numbers of GFP+ cells per microliter blood are plotted over time. (E) A section of the graph in $\mathbf{D}$ that refers to days $17-64$ is shown on a different $y$ axis scale. Means \pm SD are shown in all graphs; $n=3$ for EGFRt-high, $n=6$ for EGFRt-low, $n=2$ for mock. results, we examined the numbers of $\mathrm{CD} 45.1^{+}$cells per microliter blood over the course of the experiment (Figure 3C). We found that the numbers of EGFRt-low and EGFRt-high cells were dramatically decreased in response to cetuximab treatment, while numbers of mock-transduced cells dropped to the expected memory frequencies during the contraction phase after MVA-OVA challenge. Tracking the GFP/EGFRt ${ }^{+}$cells based on GFP expression allows assessment of the depletion effect exclusively on targetable cells (Figure 3, D and E). After 1 cetuximab injection, more than $95 \%$ of GFP/EGFRt-high cells were already depleted. Strikingly, the second dose of cetuximab eliminated any residual GFP/EGFRt-high cells within 24 hours. In contrast, depletion of GFP/EGFRt-low cells was not yet complete 24 hours after the second dose of cetuximab and followed a slower depletion kinetic over time.

These experiments demonstrate that the kinetics of cetuximab-mediated cell depletion depends on the expression level of the targeted EGFRt. T cells with high expression of EGFRt are effectively depleted within a few days after cetuximab application.
Murine CD19 CAR T cells mediate sustained B cell depletion that is permanently reversed by cetuximab administration. The potent antitumor activity of CD19 CAR T cells in patients is associated with long-term B cell aplasia. Recently, a syngeneic model of B cell leukemia in immunocompetent mice has been established, where transfer of murine T cells expressing an anti-mouse CD19 CAR (mCD19 CAR) not only effectively eradicated tumor cells, but also depleted endogenous B cells in blood, spleen, and bone marrow for at least 2 months (longer time points were not analyzed in this study) (17). To address whether depletion of CD19 CAR T cells by targeting of the EGFRt would be sufficient to abrogate $\mathrm{T}$ cell-mediated $\mathrm{B}$ cell aplasia in a clinically relevant mouse model, we introduced EGFRt into the same mCD19 CAR gene construct (m1928E), separating the transgene from EGFRt with a P2A linker to provide simultaneous expression of the CAR and EGFRt (Supplemental Figure 3A). We transduced Thy $1.1^{+}$mouse splenocytes with the m1928E construct and transferred $1.2 \times 10^{6}$ cells into sublethally irradiated WT C57BL/6 mice (Figure 4A). We 
A

WT Thy1.2 BL/6 mice are irradiated (6 Gy)

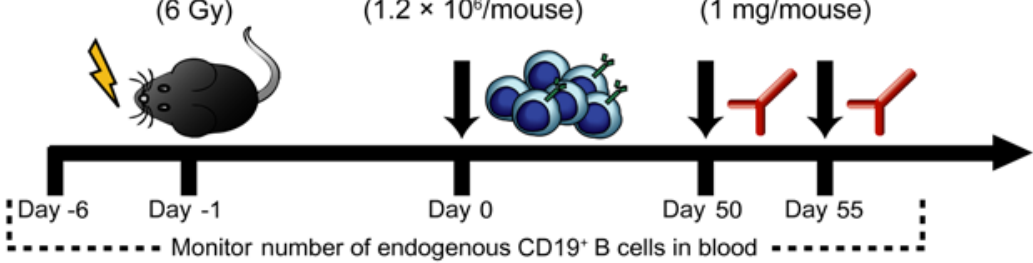

B

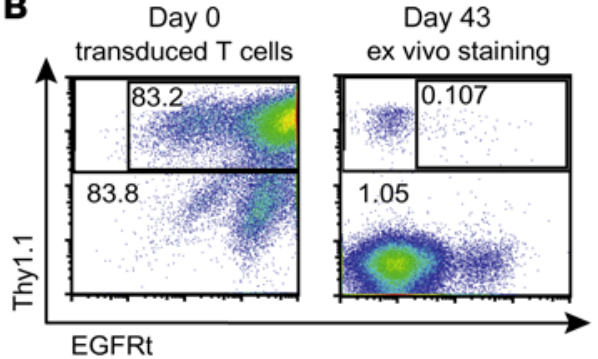

C

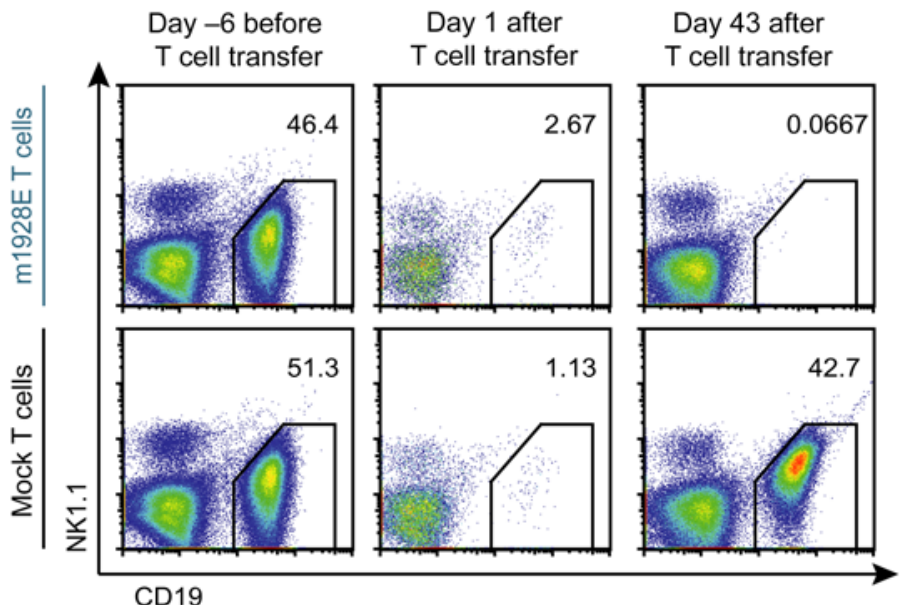

D

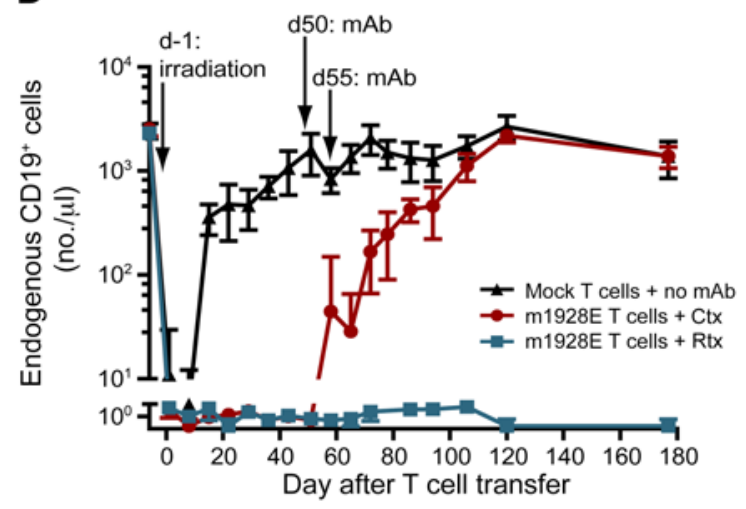

E

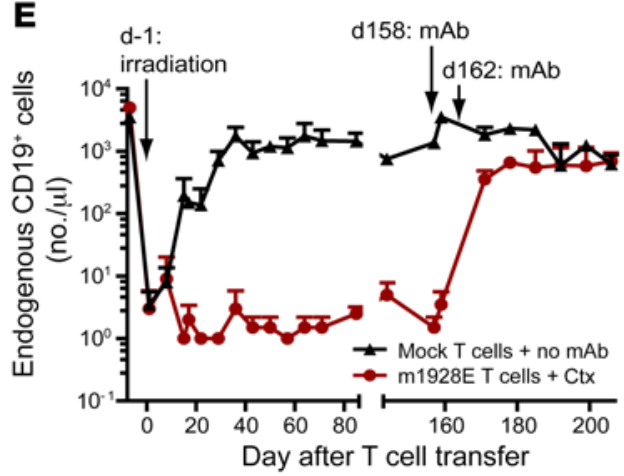

$\mathbf{F}$ IgG level on day 7 after immunization

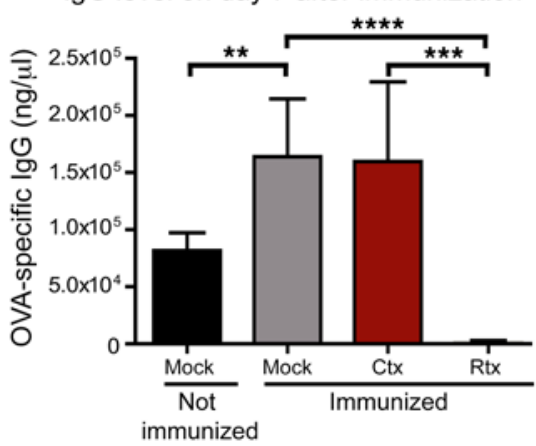

G IgG level on day 14 after immunization

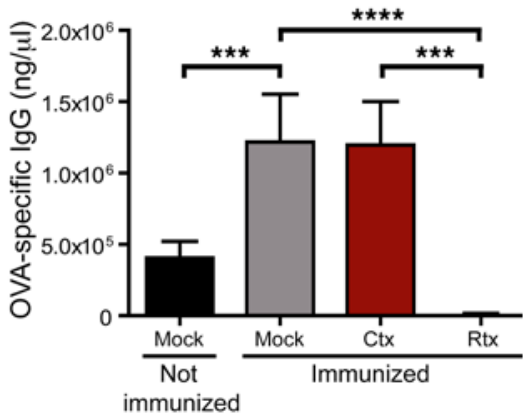

Figure 4. Cetuximab-mediated in vivo depletion of $\mathbf{m} 1928 \mathrm{E}^{+} \mathbf{T}$ cells allows for $B$ cell recovery in vivo. (A) Experimental layout of depletion experiments based on the transfer of m1928E-transduced cells targeting endogenous CD19+ B cells. (B) Thy1.1+ mouse splenocytes were retrovirally transduced with the m1928E CAR construct. After transfer of $1.2 \times 10^{6} \mathrm{~m} 1928 \mathrm{E}$-transduced (unsorted) T cells, EGFRt ${ }^{+}$cells were detected by ex vivo EGFR staining in a blood sample on day 43 after infusion. (C) Mice were irradiated on day -1 and received $m 1928 \mathrm{E}^{+} \mathrm{T}$ cells or mock $\mathrm{T}$ cells on day 0 . Numbers of endogenous $\mathrm{CD} 19^{+}$ $B$ cells were monitored in the blood before T cell transfer (day -6) and 1 or 43 days after T cell transfer. Endogenous B cells and NK cells were detected in representative samples for each group with anti-CD19 and anti-NK1.1 mAbs, respectively. Gates were set on CD19+ B cells among Thy1.1- living lymphocytes, and numbers indicate B cell frequencies. (D) Numbers of endogenous CD19+ $B$ cells per microliter blood were acquired for each mouse group over the time course. Time points of irradiation and the 2 mAb infusions are indicated. Means \pm SD are shown; $n=6$ per group. Two-way ANOVA followed by Bonferroni post-test was used for statistics. (E) In a separate long-term experiment, $8 \times 10^{6} \mathrm{~m} 1928 \mathrm{E}$-transduced, unsorted Thy1.1 $1^{+}$cells were transferred into irradiated WT C57BL/6 mice. Cetuximab infusions were performed at late time points (days 158 and 162). Numbers of endogenous CD19+ $B$ cells were obtained over the course of the experiment as described in $\mathbf{C}$ and $\mathbf{D}$. Means $\pm S D$ are plotted. $n=2$ per group. ( $\mathbf{F}$ and $\mathbf{G})$ After recovery of normal $B$ cell numbers, mice were immunized with OVA protein (100 $\mu \mathrm{g} /$ mouse) mixed 1:1 with Imject alum on day 245 after CAR T cell infusion. One mouse group had received mock T cells and was not immunized as a negative control. OVA-specific IgG levels in the blood plasma were measured by ELISA 7 days (F) and 14 days (G) after immunization. Means \pm SD are shown for 2 independent experiments; $P$ values were calculated by unpaired 2-tailed Student's $t$ test; $n=5$ for immunized mock, Ctx, and Rtx groups; $n=7$ for not immunized mock group. ${ }^{* *} P \leq 0.01,{ }^{* *} P \leq 0.001,{ }^{* * *} P \leq 0.0001$.

observed sustained B cell depletion in mice that received m1928E CAR T cells, whereas control mice that received identical numbers of mock-transduced $\mathrm{T}$ cells had rapid recovery of B cell numbers to normal levels after temporary irradiation-induced lymphopenia
(Figure 4, C and D). Ongoing B cell aplasia was associated with markedly reduced levels of IgG, IgM, and IgA in the first month after $\mathrm{T}$ cell transfer (Supplemental Figure 4). Interestingly, despite functional persistence of mCD19 CAR T cells, only low levels of 


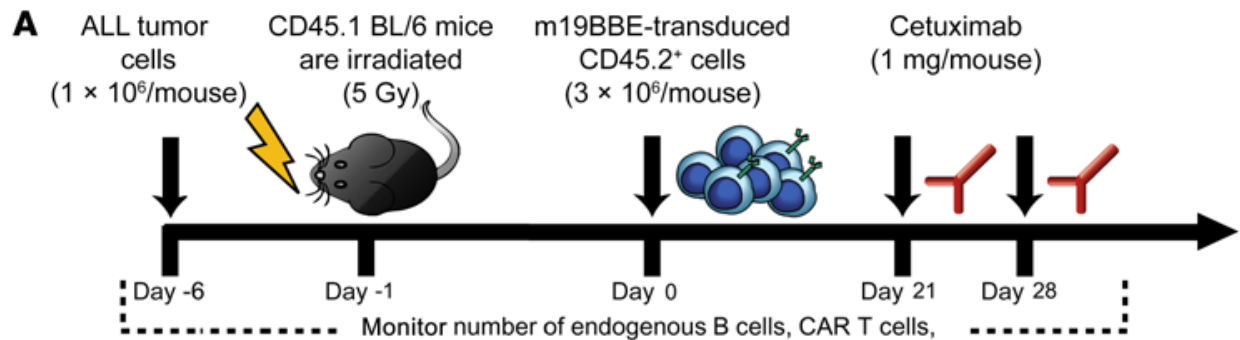
and tumor cells in blood

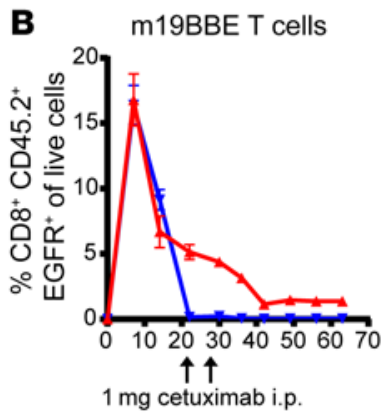

Days after $T$ cell transfer

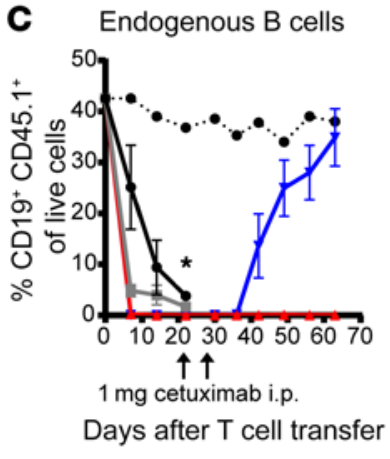

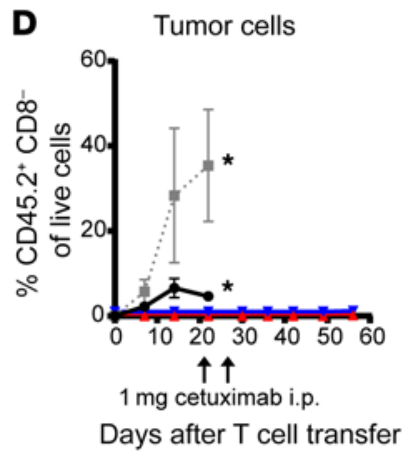

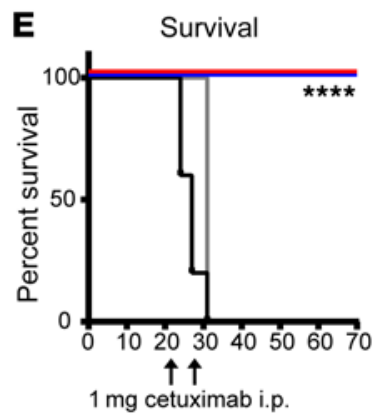

Days after tumor inoculation
F

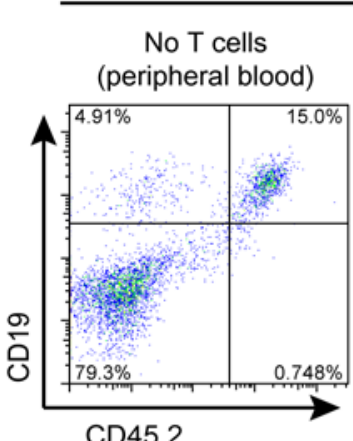

CD45.2
ALL tumor-bearing

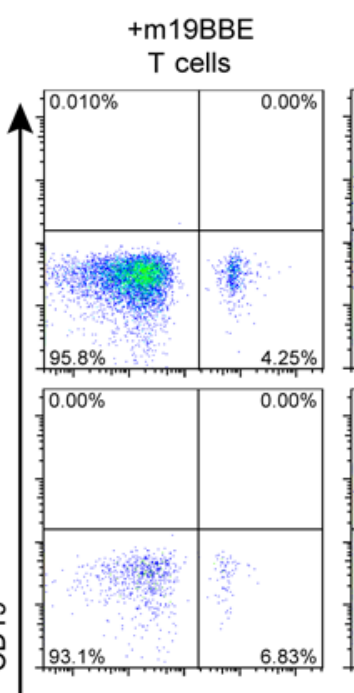

+ m19BBE T cells + cetuximab
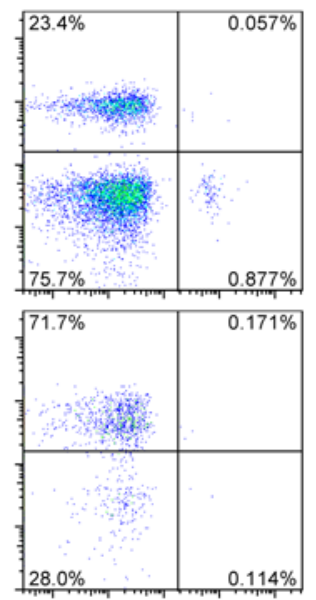

Non-tumor-bearing

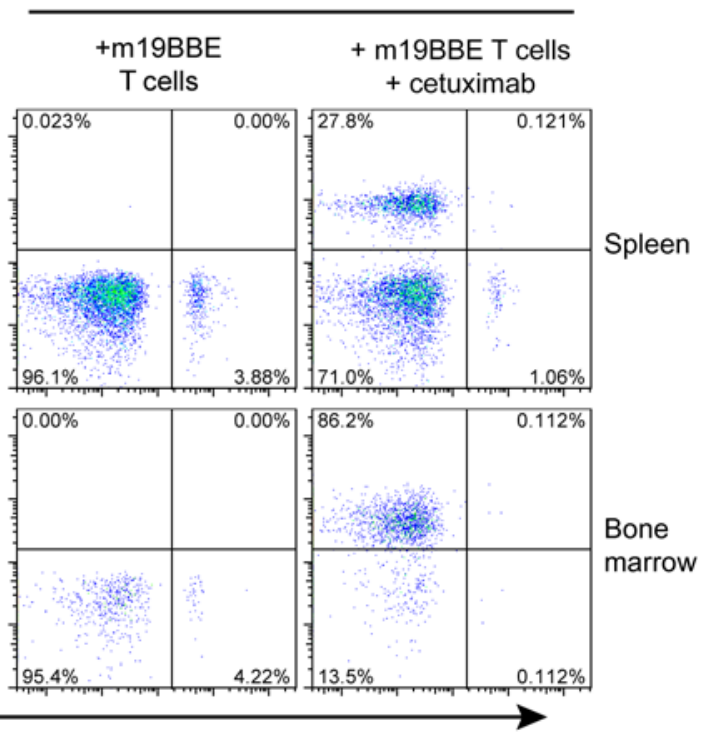

Figure 5. Cetuximab-mediated in vivo depletion of m19BBE+ murine $T$ cells does not increase tumor relapse in a model of B cell ALL. (A) Experimental layout of depletion experiments based on the transfer of m19BBE-transduced cells targeting CD19+ ${ }^{+} \mathrm{LLL}$ tumor cells. Mice were inoculated with $1 \times 10^{6} \mathrm{ALL}$ tumor cells i.v.; 6 days later they were sublethally irradiated ( $5 \mathrm{~Gy}$ ), and 24 hours later they received $3 \times 10^{6} \mathrm{CD} 8^{+}$m19BBE CAR T cells. Cohorts of mice were left untreated or treated with 2 doses of $1 \mathrm{mg}$ cetuximab on day 21 and day 28 and bled weekly to monitor T cell, normal B cell, and tumor cell frequency. (B-D) Summary of flow cytometry analysis of CD45.2+CD8+EGFR+ m19BBE mouse T cell frequency (B), CD45.1 + $^{+}$D19 ${ }^{+}$endogenous normal B cell frequency (C), and CD45.2+CD19+ tumor cell frequency (D) in the peripheral blood of mice with ALL tumors left untreated (black), mice irradiated with 5 Cy (gray), mice irradiated and treated with m19BBE CAR T cells (red), or mice irradiated and treated with m19BBE CAR T cells followed by cetuximab depletion (blue). Non-tumor-bearing WT mice are shown for comparison (dotted black line). Means \pm SEM are plotted. $n=6$ per group. (E) Kaplan-Meier survival analysis of mice inoculated with ALL tumor cells and left untreated (black), irradiated with $5 \mathrm{~Gy}$ (gray), treated with m19BBE T cells (red), or treated with m19BBE T cells and cetuximab (blue). Statistical significance was determined using log-rank Mantel-Cox test; ${ }^{*} P \leq 0.05,{ }^{* * *} P \leq 0.0001$. $n=6$ per group. (F) Representative flow cytometry data from peripheral blood of ALL tumor-bearing mice (left) 2 weeks after tumor inoculation and spleens and bone marrow of m19BBE/cetuximab-treated mice (middle) or WT non-tumor-bearing mice (right) 10 weeks after T cell transfer. Plots are gated on live CD19+ cells.

m1928E-expressing T cells were detectable in peripheral blood (Figure 4B). Next we administered 2 doses of cetuximab to a subgroup of mice that demonstrated sustained B cell depletion for 50 days after mCD19 CAR T cell treatment (Figure 4D). B cell apla- sia started to revert shortly after antibody treatment, and within 7 weeks B cell numbers reached levels similar to those in control mice that had received mock-transduced $\mathrm{T}$ cells. Importantly, mCD19 CAR T cell-treated mice that received rituximab, which 
is specific for human CD20, as control antibody did not exhibit $B$ cell recovery. The reappearance of B cell numbers after cetuximab treatment was associated with normalization of immunoglobulin levels (Supplemental Figure 4). In order to test functionality of the re-formed B cell compartment more stringently, we vaccinated mCD19 CAR T cell-treated mice after cetuximab-mediated B cell recovery with OVA in alum. As shown in Figure 4, F and G, OVA-specific IgG antibody titers reached similar levels after in vivo depletion of mCD19 CAR T cells as compared with control mice that received mock-transduced $\mathrm{T}$ cells; mice treated with rituximab, which did not affect B cell aplasia and persistence of mCD19 CAR T cells, failed to mount detectable OVA-specific antibody responses to vaccination. These data demonstrate that in vivo $\mathrm{mCD} 19$ CAR T cell depletion via targeting of coexpressed EGFRt with cetuximab can revert numerical and functional B cell deficiency, including vaccine-induced antibody generation and isotype switching.

In a clinical setting, CD19 CAR T cell depletion might be considered at even later time points after adoptive cell therapy to ensure complete tumor eradication. Therefore, we tested whether $B$ cells could be restored by cetuximab treatment when CD19 CAR T cell activity was sustained long term. B cell aplasia persisted long term (we analyzed cohorts of mice up to day 252 upon mCD19 CAR T cell treatment), despite only very low numbers of EGFRt ${ }^{+}$ $\mathrm{T}$ cells circulating in the blood and residing in the spleen or lymph nodes. A somewhat more prominent but still very small population of EGFRt ${ }^{+} \mathrm{T}$ cells was detectable in bone marrow, the site where $\mathrm{CD}_{19}{ }^{+} \mathrm{B}$ cells are first produced from stem cell progenitors (Supplemental Figures 5 and 6). We then tested whether cetuximab infusion could eliminate these low levels of CAR T cells that sustained B cell aplasia, and result in restoration of $\mathrm{B}$ cell numbers. Indeed, we observed that B cell numbers increased to normal levels in long-term CD19 CAR T cell-mediated B cell-depleted mice with kinetics that was virtually identical to that of B cell recovery when cetuximab was administered at earlier time points after CD19 CAR T cell treatment (Figure 4E).

Elimination of murine CD19 CAR T cells by cetuximab administration does not increase tumor relapse in a model of B cell $A L L$. Eliminating $\mathrm{T}$ cells engineered with a tumor-reactive CAR by administering cetuximab may increase the risk of subsequent recurrence of the malignancy. We evaluated whether elimination of mCD19 CAR $T$ cells increased the risk of relapse in mice bearing the rapidly progressive B cell leukemia E $\mu$ ALLO1, which is radioresistant in vitro. In these experiments we used an $\mathrm{mCD} 19 / 4-1 B B \zeta / E G F R t$ (m19BBE) CAR, because we and others achieved a very high rate of complete remission in clinical trials using a human CD19/ $4-1 \mathrm{BB} \zeta \mathrm{CAR}(2,18)$. Cohorts of CD $45.1^{+} \mathrm{C} 57 \mathrm{BL} / 6$ mice were inoculated i.v. with $1 \times 10^{6}$ congenically marked (CD45.2 $2^{+}$E $\mu$ ALL01 tumor cells; 6 days later, mice were sublethally irradiated (5 Gy) and were either left untreated or treated 24 hours later with $3 \times 10^{6}$ congenic CD $45.2^{+} \mathrm{CD}^{+} \mathrm{T}$ cells that expressed the m19BBE CAR (Supplemental Figure 3B). Three weeks after CAR T cell administration, a cohort of mice received 2 doses of cetuximab administered 1 week apart (Figure 5A). The CAR T cells, which had expanded in the blood, reaching a peak at 7 days after cell transfer, became undetectable in mice treated with cetuximab (Figure 5B). These mice recovered normal endogenous $\mathrm{CD} 19^{+} \mathrm{B}$ cell numbers, whereas mice not treated with cetuximab had persisting CAR T cells and remained B cell-deficient (Figure 5, B and C). Leukemia cells were readily detected in blood in mice that did not receive CAR T cells, and these mice all died from progressive leukemia in 27-31 days (Figure 5, D and E). The cetuximab-treated mice remained leukemia-free, and their survival was equivalent to that of mice that had not received cetuximab (Figure 5E). Analysis of the spleen and bone marrow using sensitive flow cytometry to stain the congenic CD $45.2^{+}$marker expressed on E $\mu$ ALLO1 tumor cells failed to detect residual leukemia cells in mice treated with cetuximab, with tumor cell frequency below the level of detection and similar to levels seen in non-tumor-bearing mice (Figure $5 \mathrm{~F}$ ). These data show that in this model of B cell ALL, the elimination of mCD19-CAR T cells can reverse B cell depletion without increasing leukemia relapse.

In conclusion, these data demonstrate that cetuximab-mediated depletion of EGFRt-coexpressing MCD19 CAR T cells efficiently reverses B cell aplasia in vivo, and provides for sustained recovery of B cell function, including normalization of serum immunoglobulin levels and the induction of isotype-switched antigen-specific antibodies upon vaccination.

\section{Discussion}

Strategies to improve the function of the transgenic tumor-targeting receptors and the long-term survival of transferred $\mathrm{T}$ cells are being evaluated in preclinical models and in clinical trials (19). Transfer of increasingly potent $\mathrm{T}$ cells is inevitably correlated with a higher risk of long-lasting adverse effects, revealing the need for an effective approach to eliminate $\mathrm{T}$ cells that can mediate on-target or off-target toxicities. The expression of a conditional suicide gene in the transferred $\mathrm{T}$ cells would be an ideal strategy controlling their survival after adoptive transfer. Prior studies have shown that introducing the herpes simplex virus thymidine kinase (HSV-TK) gene into allogeneic $\mathrm{T}$ cells renders dividing cells sensitive to ganciclovir, and administering this drug was effective for reversing GVHD resulting from donor lymphocyte infusions used to treat relapse after allogeneic hematopoietic stem cell transplant (11). Unfortunately, HSV-TK is immunogenic in humans, and the development of $\mathrm{T}$ cell immunity to the viral protein can lead to elimination of transferred $\mathrm{T}$ cells that do not cause toxicity (9). Conditional suicide genes based on activation of chimeric human death receptors composed of portions of CD95 (FAS) or caspase-9 fused to 2 copies of an FKBP variant with a point mutation to enhance binding of a synthetic dimerizer drug have also been developed (20-23). Inducible caspase-9 (iCasp9) has been used successfully to eliminate allogeneic T cells that cause GVHD after haploidentical hematopoietic stem cell transplant (8). The iCasp9 construct contains unique fusion sites and a point mutation that have the potential to be immunogenic in less immunosuppressed individuals. We developed the human EGFRt cell surface marker that can be targeted by cetuximab for in vivo depletion of engineered cells. Here we show, for the first time to our knowledge, that a long-term functional deficiency of normal B cells caused by the adoptive transfer of CD19 CAR T cells can be reversed by $\mathrm{mAb}$-mediated in vivo depletion of the $\mathrm{T}$ cells that express the EGFRt along with a CD19 CAR.

We first coexpressed the EGFRt with GFP in OT-I TCR transgenic $\mathrm{T}$ cells using retroviral gene delivery and found that EGFRt 
is stably expressed for more than 150 days after adoptive transfer, which is important in situations where toxicity might necessarily be tolerated to allow tumor eradication to be complete, but still be reversible at late times after $\mathrm{T}$ cell transfer. Our studies showed that the administration of cetuximab eliminated $\mathrm{T}$ cells with high levels of EGFRt more rapidly and efficiently than T cells that expressed low levels of EGFRt. This result is consistent with studies demonstrating that cetuximab-mediated antibody-dependent cellular cytotoxicity is more efficient against target cells that express high levels of EGFR (24), and emphasizes the importance of using vector designs, such as those employing ribosomal skip elements, where EGFRt is coexpressed with the tumor-targeting receptor $(16,25)$. This also suggests that selecting the subset of $\mathrm{T}$ cells that express high levels of EGFRt prior to transfer will ensure efficient ablation after adoptive therapy. EGFRt can be used for enrichment of transduced $\mathrm{T}$ cells prior to transfer, and clinical selection reagents and procedures have been developed to preferentially purify the cell population that expresses high levels of the EGFRt and coexpressed CAR for clinical applications (16).

To study the potential to target EGFRt to reverse long-term T cell-mediated toxicities, we used a syngeneic mouse model based on transfer of murine T cells that coexpress a mouse CD19-specific CAR and EGFRt. In this model, the administration of mCD19 CAR T cells induced long-term depletion of endogenous B cells that was sustained by low numbers of CAR T cells that persisted in the blood (about 5 CAR T cells per microliter), and resulted over time in a profound reduction in serum immunoglobulin levels, similar to what has been reported in patients receiving CD19 CAR $\mathrm{T}$ cells $(5,26)$. Both early and late administration of cetuximab depleted CD19 CAR T cells in vivo and was effective in reversing $B$ cell aplasia. B cell numbers recovered to normal levels by 7 weeks after cetuximab administration and remained stable, demonstrating that any persisting CAR T cells were not able to reinduce the $B$ cell deficiency, perhaps because they expressed insufficient levels of the CAR to recognize $\mathrm{CD} 19^{+} \mathrm{B}$ cells. The B cells that recovered expressed normal levels of CD19 and were functional as demonstrated by the ability of the mice to mount IgM and IgG responses to an OVA immunization. Thus, this mouse model could provide new insights into the kinetics of recovery of B cell function and reversal of hypogammaglobulinemia.

The EGFRt transgene has been incorporated into clinical CD19 CAR vectors that are currently being administered to patients with B cell malignancies with no signs of immunogenicity directed against the EGFRt component of the vector. Cetuximab is available as a clinical-grade antibody, and its side effects are limited to grade 1 or 2 skin rash in $86 \%$ of patients and occasional severe skin rash (grade 3 or 4 ) in 10\%-15\% of patients (27). Cetuximab does not cross-react with murine EGFR; thus our studies could not determine whether the use of the antibody to eliminate EGFRt ${ }^{+}$ $\mathrm{T}$ cells might unexpectedly affect its toxicity profile. The data presented here support testing whether administration of cetuximab can recover the B cell compartment in patients with complete tumor regression, persistent CD19 CAR T cells, and B cell aplasia. A challenge will be to decide when to eliminate the CAR $\mathrm{T}$ cells, since it is not yet known how long T cells must be present to ensure that patients will not relapse. Highly sensitive techniques for molecular monitoring of clonal IgH rearrangements in $\mathrm{B}$ cell malignancies are useful for predicting relapse after chemotherapy and might be used similarly to identify patients with a low risk of relapse after CAR T cell therapy (28). Our data in a murine B cell ALL model show that CAR T cells can be depleted 1 month after adoptive transfer, allowing the recovery of normal B cells without leukemia relapse. This should not be taken to conclude that elimination of CAR T cells in patients will not increase relapse, since a variety of factors, including type of malignancy, tumor burden, and susceptibility to $\mathrm{T}$ cell eradication, may determine the risk of relapse. Even if relapse occurred after CAR T cell ablation, it could potentially be managed by a repeated infusion of CAR T cells, after the cetuximab is cleared.

A potential limitation of this depletion strategy is that it may not be sufficiently rapid to reverse toxicity resulting from cytokines released by activated $\mathrm{T}$ cells or to reverse serious toxicity to cells that cannot be easily replenished. We did observe efficient reduction of $\mathrm{T}$ cell numbers within 24 hours after the second $\mathrm{mAb}$ infusion, including $\mathrm{T}$ cells residing in secondary lymphoid organs or other vital organs, which may be the site of unexpected acute adverse effects $(29,30)$. The rapidity of $\mathrm{T}$ cell elimination might be improved by enhancing the Fc portion of anti-EGFR mAbs to improve antibody-dependent cellular cytotoxicity; thus it is conceivable that EGFRt could be used to rapidly counteract even acute side effects.

In summary, we demonstrated that the EGFRt marker is stably expressed on $\mathrm{T}$ cells and can be targeted with cetuximab to eliminate CAR-modified $\mathrm{T}$ cells after adoptive transfer. This safety strategy can be integrated in genetically engineered $\mathrm{T}$ cells that target other tumor-associated molecules and pathogens, and may therefore be broadly applied to enhance control over highly potent cell therapeutics.

\section{Methods}

Mice and cell lines. C57BL/6 mice were purchased from Harlan Laboratories (Harlan Winkelmann, Borchen). C57BL/6 CD45.1 congenic mice were purchased from The Jackson Laboratory. Female OT-I C57BL/6 mice were derived from in-house breeding under specific pathogen-free conditions. The $\mathrm{E} \mu$ ALL01 cell line was derived from an E $\mu$-myc transgenic mouse as previously described (17).

Antibodies and reagents. The human biotinylated EGFR-specific mAb was generated as described previously (16). The biotinylated human Fc-specific Fab reagent was purchased from Jackson ImmunoResearch. Fluorochrome-labeled antibodies specific to mouse CD45.1 (clone A20), mouse Thy1.1 (clone HIS51), mouse CD8 (clone 53-6.7), mouse CD45.2 (clone 104), and mouse NK1.1 (clone PK136) were obtained from eBioscience; the anti-mouse CD19 antibody (clone 1D3) and streptavidin were purchased from BD Pharmingen. The EGFR-specific Fab Streptamer was obtained from IBA. Purified antimouse CD3 (clone 145-2c11) and anti-mouse CD28 (clone 37.51), both purchased at BD Pharmingen, were used for activating mouse splenocytes before transductions. Cetuximab (Bristol-Myers Squibb) and rituximab (Genentech) were used for antibody injections in the mouse depletion studies.

Retroviral vector construction. Retroviral vector plasmid pMIGR1 or pMP71 was used to synthesize all vectors used in this study.

Cell culture and transduction of T cells. Murine T cells were cultivated in RPMI 1640 (PAA Laboratories), supplemented with 10\% FCS, 
0.025\% L-glutamine, 0.1\% HEPES, 0.001\% gentamycin, $0.002 \%$ streptomycin, and $100 \mathrm{U} / \mathrm{ml}$ of IL-2 (Proleukin S, Novartis). Platinum-E cells were grown in DMEM (PAA Laboratories), supplemented with $10 \%$ FCS, $0.025 \%$ L-glutamine, 0.1\% HEPES, 0.001\% gentamycin, and $0.002 \%$ streptomycin.

For transduction, murine splenocytes were obtained from OT-I transgenic or WT C57BL/6 mouse spleens, brought into single-cell suspension, and incubated in erythrocyte lysis buffer once. Mouse splenocytes were stimulated overnight with purified anti-mouse CD3 and anti-mouse CD28 antibodies at a dilution of 1:500 or with antiCD3/anti-CD28-coated beads. For retrovirus production, Platinum-E packaging cells were transiently transfected with the retroviral vector plasmids using Effectene (Qiagen) according to the manufacturer's manual. The supernatant of the Platinum-E cells was collected after 48 and 72 hours, filtered through 0.45 - $\mu \mathrm{m}$-pore filters, and used to transduce cells on 2 consecutive days. Platinum-E supernatant, supplemented with $100 \mathrm{U} / \mathrm{ml} \mathrm{IL-2}$ and $4 \mu \mathrm{g} / \mathrm{ml}$ protamine sulfate (SigmaAldrich), was spinoculated onto plates coated with RetroNectin (Takara Bio Europe SAS) at 2,000 $g$ at $32^{\circ} \mathrm{C}$ for 90 minutes. Stimulated mouse splenocytes were added to the plates and centrifuged at $1,000 \mathrm{~g}$ at $32^{\circ} \mathrm{C}$ for 10 minutes.

Flow cytometry staining and sorting. Cells were stained with the respective antibody panel in the dark at $4^{\circ} \mathrm{C}$ for 20 minutes. For staining murine cells, Fc block was added at a dilution of 1:400. Propidium iodide (Invitrogen) was used for live/dead discrimination. Samples were acquired on a CyAn AD Lx flow cytometer (Dako Cytomation) and analyzed using FlowJo software (Tree Star). For sorting, transduced mouse cells were stained with EGFR-specific Streptamers according to the manufacturer's protocol. Staining with anti-mouse CD19 mAb and propidium iodide was added to allow for exclusion of B cells and dead cells. Sorting of live $\mathrm{GFP}^{+}, \mathrm{EGFRt}^{+}$cells was performed on a MoFloII cell sorter (Dako Cytomation). To remove EGFR Fab Streptamer from the cell surface, sorted cells were incubated in $1 \mathrm{mM}$ D-biotin at room temperature for 10 minutes and washed in a large volume of buffer twice.

Adoptive transfer of $T$ cells and in vivo depletion studies. One day before cell transfer, C57BL/6 mice were sublethally $\gamma$-irradiated (5 or $6 \mathrm{~Gy}$ ). Mouse OT-I cells that had been transduced with GFP/EGFRt or mCD19CAR/EGFRt retroviral constructs, and sort-purified for EGFRt ${ }^{+}$cells where indicated, were transferred into mice by i.v. injection. To expand OT-I cells in vivo, mice were vaccinated by i.v. application of a modified vaccinia virus Ankara-based vector encoding ovalbumin (MVA-OVA) at $1 \times 10^{8}$ PFU per mouse (12). For depletion of transferred EGFRt ${ }^{+} \mathrm{T}$ cells, cetuximab or rituximab as a control mAb was infused i.p. at $1 \mathrm{mg}$ per mouse at specified time points. Expansion and depletion of transferred cells were monitored in blood samples by flow cytometry. At the end of experiments, spleens, lymph nodes, bone marrow, and/or lungs were obtained in addition to blood to assess persistence and depletion of transferred cells in lymphoid and peripheral organs.

To assess immunoglobulin levels, blood plasma was obtained by centrifuging of heparinized blood at 8,000 $\mathrm{g}$ for 5 minutes and collection of the supernatant. In vivo B cell function was tested by vaccination of mice i.p. with OVA (100 $\mu \mathrm{g} /$ mouse) mixed 1:1 with Imject alum (Thermo Fisher Scientific Inc.). The OVA-specific IgG levels were analyzed on day 7 and day 14 after immunization.

Detection of anti-OVA specific antibody responses by ELISA. Mice were bled from the tail vein at days 7 and 14 after immunization. The titers of anti-OVA antibodies were determined by ELISA as described previously (31), with minor modifications. Briefly, 96-well plates (Nunc MaxiSorp ELISA Plates, Uncoated, Thermo Fisher Scientific Inc.) were coated with $200 \mu \mathrm{l}$ of $100 \mu \mathrm{g} / \mathrm{ml}$ OVA protein (SigmaAldrich) with $0.5 \mathrm{M}$ carbonate buffer saline (0.159 $\mathrm{g}$ of $\mathrm{Na}_{2} \mathrm{CO}_{3}$ and $0.293 \mathrm{~g}$ of $\mathrm{NaHCO}_{3}$ dissolved in $100 \mathrm{ml}$ of double-deionized $\mathrm{H}_{2} \mathrm{O}, \mathrm{pH}$ 9.6 , overnight at $4^{\circ} \mathrm{C}$ ). Wells were washed and blocked with $5 \%$ BSA in $1 \times$ Tris-Tween-buffered saline for 2 hours at $37^{\circ} \mathrm{C}$ and washed, and then $100 \mu \mathrm{l}$ sera from immunized mice (triplicates) were added to the wells at different dilutions. After incubation and washing, biotin-conjugated rabbit anti-mouse IgG (1:5,000; Sigma-Aldrich) was used as a secondary antibody. For detection, HRP-conjugated streptavidin was added for 1 hour at room temperature (1:1,000; Sigma-Aldrich). Plates were incubated and washed, and the reaction was developed by addition of $3,3^{\prime}, 5,5^{\prime}$-tetramethylbenzidine (TMD, Pierce) substrate solution and stopped by addition of $100 \mu \mathrm{l} /$ well of $0.2 \mathrm{M} \mathrm{H}_{2} \mathrm{SO}_{4}$. The microplates were read at $450 \mathrm{~nm}$ (Sunrise, Tecan). The cutoff was determined as the titer of preimmune sera at the same dilution. For determination of endpoint titers, sera were serially diluted up to 1:1,024,000 from an initial dilution of 1:1,000 and calculated with a standard curve as described.

Statistics. All statistical analysis was performed with GraphPad Prism 6 for Windows, version 6.05 (GraphPad Software). Student's $t$ test was used to compare 2 groups. Multiple groups were analyzed by 2-way ANOVA, followed by Bonferroni post-test. Level of significance was calculated with the unpaired Mann-Whitney test or unpaired 2-tailed Student's $t$ test as indicated. When multiple groups at multiple time points were compared, Student's $t$ test (2-tailed) or ANOVA for each time point was used. Survival curves were compared using the log-rank MantelCox test. Kaplan-Meier plots were generated using GraphPad Prism, and $P$ values for curve comparisons were calculated using the log-rank Mantel-Cox method. Significance was defined as $P$ less than 0.05.

Study approval. All experiments were performed in accordance with the guidelines of the Institutional Animal Care and Use Committee of the Fred Hutchinson Cancer Research Center (IR 1806) or the Regierung von Oberbayern (AZ 55.2-1-54-2532-158-12).

\section{Author contributions}

DHB and SRR provided intellectual input and the conceptual framework and helped to design experiments and to write the manuscript. PJP, SPF, and SS designed and performed experiments, collected and analyzed data, and wrote the manuscript. DS, MH, ID, MS, LL, and MCJ provided biological material.

\section{Acknowledgments}

This work was supported by SFB TR36 (TP-B10/13), SFB 1054 (TP-B09), and GRK 1949 A1 from the DFG, as well as NIH grants CA136551, CA114536, P30 CA008748, and P50 CA138293.

Address correspondence to: Dirk H. Busch, Institute for Medical Microbiology, Immunology and Hygiene, Technische Universität München, Trogerstraße 30, D-81675 Munich, Germany. Phone: 49.89.4140.4120; E-mail: dirk.busch@tum.de. Or to: Stanley R. Riddell, Program in Immunology, Fred Hutchinson Cancer Research Center, 1100 Fairview Avenue N., Seattle, Washington 98109-1024, USA. Phone: 206.667.5249; E-mail: sriddell@fhcrc.org. 
1. Kochenderfer JN, et al. Chemotherapy-refractory diffuse large B-cell lymphoma and indolent B-cell malignancies can be effectively treated with autologous $\mathrm{T}$ cells expressing an anti-CD19 chimeric antigen receptor. JClin Oncol. 2015;33(6):540-549.

2. Maude SL, et al. Chimeric antigen receptor T cells for sustained remissions in leukemia. $N$ Engl JMed. 2014;371(16):1507-1517.

3. Davila ML, et al. Efficacy and toxicity management of 19-28z CAR T cell therapy in B cell acute lymphoblastic leukemia. Sci Transl Med. 2014;6(224):224ra25.

4. Pontvert-Delucq S, et al. Characterization and functional analysis of adult human bone marrow cell subsets in relation to B-lymphoid development. Blood. 1993;82(2):417-429.

5. Kalos M, et al. T cells with chimeric antigen receptors have potent antitumor effects and can establish memory in patients with advanced leukemia. Sci Transl Med. 2011;3(95):95ra73.

6. Gilham DE, Debets R, Pule M, Hawkins RE, Abken H. CAR-T cells and solid tumors: tuning $\mathrm{T}$ cells to challenge an inveterate foe. Trends $\mathrm{Mol}$ Med. 2012;18(7):377-384.

7. Ciceri F, et al. Infusion of suicide-gene-engineered donor lymphocytes after family haploidentical haemopoietic stem-cell transplantation for leukaemia (the TK007 trial): a non-randomised phase I-II study. Lancet Oncol. 2009;10(5):489-500.

8. Di Stasi A, et al. Inducible apoptosis as a safety switch for adoptive cell therapy. $N$ Engl J Med. 2011;365(18):1673-1683.

9. Berger C, Flowers ME, Warren EH, Riddell SR. Analysis of transgene-specific immune responses that limit the in vivo persistence of adoptively transferred HSV-TK-modified donor $\mathrm{T}$ cells after allogeneic hematopoietic cell transplantation. Blood. 2006;107(6):2294-2302.

10. Lee WY, Zhang T, Lau CP, Wang CC, Chan KM, Li G. Immortalized human fetal bone marrow-derived mesenchymal stromal cell expressing suicide gene for anti-tumor therapy in vitro and in vivo. Cytotherapy. 2013;15(12):1484-1497.

11. Bonini C, et al. HSV-TK gene transfer into donor lymphocytes for control of allogeneic graft-versusleukemia. Science. 1997;276(5319):1719-1724.

12. Graef $P$, et al. Serial transfer of single-cellderived immunocompetence reveals stemness of CD8(+) central memory T cells. Immunity. 2014;41(1):116-126.

13. Vogler I, et al. An improved bicistronic CD2O/ tCD34 vector for efficient purification and in vivo depletion of gene-modified $\mathrm{T}$ cells for adoptive immunotherapy. Mol Ther. 2010;18(7):1330-1338.

14. Philip B, et al. A highly compact epitope-based marker/suicide gene for easier and safer T-cell therapy. Blood. 2014;124(8):1277-1287.

15. Kieback E, Charo J, Sommermeyer D, Blankenstein $\mathrm{T}$, Uckert $\mathrm{W}$. A safeguard eliminates $\mathrm{T}$ cell receptor gene-modified autoreactive $\mathrm{T}$ cells after adoptive transfer. Proc Natl Acad Sci U S A. 2008;105(2):623-628.

16. Wang X, et al. A transgene-encoded cell surface polypeptide for selection, in vivo tracking, and ablation of engineered cells. Blood. 2011;118(5):1255-1263.

17. Davila ML, Kloss CC, Gunset G, Sadelain M. CD19 CAR-targeted T cells induce long-term remission and B cell aplasia in an immunocompetent mouse model of $\mathrm{B}$ cell acute lymphoblastic leukemia. PLoS One. 2013;8(4):e61338.

18. Turtle CJ, et al. CD19 CAR-T cells of defined $\mathrm{CD} 4^{+}: \mathrm{CD} 8^{+}$composition in adult $\mathrm{B}$ cell ALL patients. J Clin Invest. 2016;126(6):2123-2138.

19. Jensen MC, Riddell SR. Design and implementation of adoptive therapy with chimeric antigen receptor-modified T cells. Immunol Rev. 2014;257(1):127-144.

20. Berger C, et al. Pharmacologically regulated Fas-mediated death of adoptively transferred $\mathrm{T}$ cells in a nonhuman primate model. Blood. 2004;103(4):1261-1269.

21. Tey SK, Dotti G, Rooney CM, Heslop HE, Brenner MK. Inducible caspase 9 suicide gene to improve the safety of allodepleted $\mathrm{T}$ cells after haploidentical stem cell transplantation. Biol Blood Marrow Transplant. 2007;13(8):913-924.

22. Straathof KC, et al. An inducible caspase 9 safety switch for T-cell therapy. Blood. 2005;105(11):4247-4254.

23. Thomis DC, et al. A Fas-based suicide switch in human $\mathrm{T}$ cells for the treatment of graft-versushost disease. Blood. 2001;97(5):1249-1257.

24. Derer S, et al. Impact of epidermal growth factor receptor (EGFR) cell surface expression levels on effector mechanisms of EGFR antibodies. JImmunol. 2012;189(11):5230-5239.

25. Yang S, et al. Development of optimal bicistronic lentiviral vectors facilitates high-level TCR gene expression and robust tumor cell recognition. Gene Ther. 2008;15(21):1411-1423.

26. Porter DL, Levine BL, Kalos M, Bagg A, June $\mathrm{CH}$. Chimeric antigen receptor-modified T cells in chronic lymphoid leukemia. $N$ Engl J Med. 2011;365(8):725-733.

27. Peréz-Soler R, Saltz L. Cutaneous adverse effects with HER1/EGFR-targeted agents: is there a silver lining? J Clin Oncol. 2005;23(22):5235-5246.

28. Brentjens RJ, et al. CD19-targeted T cells rapidly induce molecular remissions in adults with chemotherapy-refractory acute lymphoblastic leukemia. Sci Transl Med. 2013;5(177):177ra38.

29. Morgan RA, Yang JC, Kitano M, Dudley ME, Laurencot CM, Rosenberg SA. Case report of a serious adverse event following the administration of $\mathrm{T}$ cells transduced with a chimeric antigen receptor recognizing ERBB2. Mol Ther. 2010;18(4):843-851.

30. Linette GP, et al. Cardiovascular toxicity and titin cross-reactivity of affinity-enhanced T cells in myeloma and melanoma. Blood. 2013;122(6):863-871.

31. Salgado J, Gilabert A, Castell M, Castellote C, Queralt J. ELISA for quantification of specific IgG and IgE antibodies to ovalbumin. Allergol Immunopathol (Madr). 1988;16(2):95-98. 